\title{
Limit Theorems for The Sums of Random Variables in A Special Form
}

\author{
Azam A. Imomov ${ }^{1,2, *}$, Zuhriddin A. Nazarov ${ }^{2}$ \\ ${ }^{1}$ Department of Mathematics, Faculty of Physics and Mathematics, Karshi State University, Karshi, Uzbekistan \\ ${ }^{2}$ V.I.Romanovskiy Institute of Mathematics, Academy of Sciences of Uzbekistan, Tashkent, Uzbekistan
}

Received July 27, 2021; Revised January 15, 2022; Accepted January 25, 2022

Cite This Paper in the following Citation Styles

(a): [1] Azam A. Imomov, Zuhriddin A. Nazarov, "Limit Theorems for The Sums of Random Variables in A Special Form," Mathematics and Statistics, Vol.10, No.1, pp. 262-268, 2022. DOI: 10.13189/ms.2022.100125

(b): Azam A. Imomov, Zuhriddin A. Nazarov, (2022). Limit Theorems for The Sums of Random Variables in A Special Form. Mathematics and Statistics, 10(1), 262-268. DOI: 10.13189/ms.2022.100125

Copyright $@ 2022$ by authors, all rights reserved. Authors agree that this article remains permanently open access under the terms of the Creative Commons Attribution License 4.0 International License

\begin{abstract}
In this paper, we consider some functionals of the sums of independent identically distributed random variables. The functionals of the sums are important in probabilistic models and stochastic branching systems. In connection with the application in various probabilistic models and stochastic branching systems, we are interested in the fulfillment of the law of large numbers and the Central limit theorem for these sums. The main hypotheses of the paper are the presence of second order moments of the variables and the fulfillment of the Lindeberg condition is considered. The research object and subject of this paper consists of specially generated random variables using the sums of non-bound random variables. In total, 6 different sums in a special form were studied in the paper and this sum was not previously studied by other scientists. The purpose of the paper is to examine whether these sums in a special form satisfy the terms of the law of large numbers and the Central limit theorem. The main result of the paper is to show that the law of large numbers and the terms of the classical limit theorem are fulfilled in some cases. The results obtained in the paper are of theoretical importance, The Central limit theorem analogues proved here are applications of Lindeberg theorem. The results can be applied to the determination of the fluctuation of immigration branching systems as well as the asymptotic state of autoregression processes. At the same time, from the main results obtained in the paper it can be used in practical lessons conducted on the theory of probability. The results of the paper will be an important guide for young researchers. Important theorems proved in the paper can be used in probability theory, stochastic branching systems and other practical problems.
\end{abstract}

in a Special Form, Law of Large Numbers, Central Limit Theorem, Stochastic Branching Systems

\section{Introduction}

Let $\mathbb{N}$ be set of natural numbers and $\left\{\xi_{n}, n \in \mathbb{N}\right\}-$ a sequence of random variables. The solution of important problems in many areas of probability theory and mathematical statistics leads to the determination of the asymptotic state of the sum

$$
S_{n}=\xi_{1}+\xi_{2}+\cdots+\xi_{n}
$$

as $n \rightarrow \infty$. The asymptotic behaviour of $S_{n}$ has been widely studied in probability theory, in which the law of large numbers, strong law of large numbers and Central limit theorems play an important role. Along with this, in many cases it becomes necessary to study the asymptotic behaviours of random variables in the form of the sum $\sum_{k=1}^{n} f_{n k}\left(S_{k}\right)$, where $f_{n k}$ is some functional. For example, we considering the first-order autoregressive process

$$
X_{k}=\mu X_{k-1}+\varepsilon_{k}, \quad k \in \mathbb{N}
$$

with lag coefficient $\mu$, we can rewrite this relation as an sum of a purely random process using reverse substitution as follows:

$$
X_{n}=\sum_{k=1}^{n} \mu^{n-k} \varepsilon_{k},
$$

where $\varepsilon_{k}$ are independent random variables; see [3]. 
Similarly, the population size in the Galton-Watson branching-immigration system is determined by the following recursive random sum:

$$
X_{0}=0, \quad X_{k}=\sum_{j=1}^{X_{k-1}} \xi_{k, j}+\varepsilon_{k},
$$

where $\xi_{k, j}$ and $\varepsilon_{k}$ are number of "aborigines" and number of immigrants entered the system in the time $k$, respectively; see [1] and [2]. Then the deviation of the random variable $X_{n}$ from its mean will take the form

$$
X_{n}-\mathrm{E} X_{n}=\sum_{k=1}^{n} m^{n-k} M_{k},
$$

where $m$ is the per mean capita number of "aborigines" and $M_{k}$ is martingale difference; see for instance [4]. In this model, it will be necessary to study the asymptotes of the random variables $\sum_{k=1}^{n} f_{n k}\left(M_{k}\right)$ where

$$
f_{n k}(x)=m^{n-k} x .
$$

The Chebyshev inequality (also called the BiennemChebyshev inequality), the Bernoulli and Chebyshev theorems, the Moivre-Laplace integral theorem, the Lindeberg condition, and the Lindeberg theorem are important concepts of limit theorems. The study of limit theorems for a sum of random variables is one of the main problems of probability theory and mathematical statistics. In this field, significant results have been achieved by P.L.Chebyshev, A.N.Kolmogorov, B.V.Gnedenko, A.Ya.Khinchin, W.Feller, A.V.Prokhorov, Ya.V.Lindeberg, V.M.Zolotarev and etc.; see [5], [6].

In this report, we define the condition for the applicability of the Central limit theorem for sums of a special form, which have not been studied previously. In particular, our aim is to determine the conditions under which the Central limit theorem is applicable to sums of a special form. We observe limit theorems for sums of random variables of a special form and describe the main results obtained. The main assumptions in the paper are the presence of second-order moments for the variables under consideration and the fulfillment of the Lindeberg condition. The main results obtained are of theoretical importance and can be applied to determine fluctuations of stochastic branching-immigration systems, as well as the asymptotic behavior of autoregressive processes.

\subsection{The law of large numbers and the strong law of large numbers}

In probability theory, one often has to work with the sum of a fairly large number of random variables. This sum loses its random character when the number of terms is large enough, and the terms themselves satisfy certain conditions. In probability theory there are a number of statements and theorems called limit theorems. They represent the relationship between random variables in a fairly large number of trials. Limit theorems are conventionally divided into two groups. The first group of theorems is called the laws of large numbers. These theorems state the stability of the mean of random variables. In a sufficiently large number of trials, the mean of random variables loses its randomness. The second group of theorems is called Central limit theorems. In these theorems, conditions are given under which, in a sufficiently large number of trials, the distribution of the sum of random variables converges to the normal distribution.

According to papers we give the following Theorems and Definitions about the law of large numbers and the strong law of large numbers; see [7]-[13].

Let $\left\{\xi_{n}, n \in \mathbb{N}\right\}$ be a sequence of independent and identically distributed random variables determined on the probability space $(\boldsymbol{\Omega}, \mathcal{F}, \mathbb{P})$. Put

$$
S_{n}=\sum_{i=1}^{n} \xi_{i} \quad \text { and } \quad A_{n}=\sum_{i=1}^{n} \mathrm{E} \xi_{i} .
$$

Definition 1. If for any $\varepsilon>0$ the relation

$$
\lim _{n \rightarrow \infty} \mathbb{P}\left(\left|S_{n}-A_{n}\right| \geq n \varepsilon\right)=0
$$

holds, i.e.

$$
\frac{1}{n} \sum_{k=1}^{n}\left(\xi_{k}-\mathrm{E} \xi_{k}\right) \stackrel{P}{\longrightarrow} 0 \quad \text { as } n \rightarrow \infty,
$$

then the sequence $\left\{\xi_{n}, n \in \mathbb{N}\right\}$ is said to satisfy the law of large numbers $(L L N)$. Where the symbol " $\stackrel{P}{\longrightarrow}$ " denotes the convergence in probability.

Definition 2. If

$$
\frac{S_{n}-A_{n}}{n} \longrightarrow 0 \quad \text { with prob. } 1
$$

as $n \rightarrow \infty$, that is

$$
\mathbb{P}\left(\omega: \lim _{n \rightarrow \infty} \frac{1}{n}\left|S_{n}(\omega)-A_{n}\right|=0\right)=1,
$$

then the sequence $\left\{\xi_{n}, n \in \mathbb{N}\right\}$ is said to satisfy the strong law of large numbers (SLLN).

Thus LLN, often referred as a simple or weak law of large numbers, is associated with convergence in probability, and SLLN is associated with almost sure convergence or convergence with Probability 1.

Now we present well-known classical results related to LLN and SLLN in the following Theorems 1]-3, see [7].

Theorem 1. (Khinchin). Let $\left\{\xi_{n}, n \in \mathbb{N}\right\}$ be a sequence of independent and identically distributed random variables with finite mean $a:=\mathrm{E} \xi_{n}$. Then the sequence $\left\{\xi_{n}, n \in \mathbb{N}\right\}$ satisfies $L L N$ :

$$
\frac{1}{n} \sum_{k=1}^{n} \xi_{k} \stackrel{P}{\longrightarrow} a \quad \text { as } n \rightarrow \infty .
$$

Theorem 2. (Markov). Let $\left\{\xi_{n}, n \in \mathbb{N}\right\}$ be a sequence of arbitrary random variables. If

$$
\frac{1}{n^{2}} \operatorname{Var} S_{n} \longrightarrow 0 \quad \text { as } n \rightarrow \infty,
$$

then the sequence $\left\{\xi_{n}, n \in \mathbb{N}\right\}$ satisfies $L L N$ in the sense of Definition 1$]$ 
Theorem 3. (Kolmogorov). Let for the sequence of independent random variables $\left\{\xi_{n}, n \in \mathbb{N}\right\}$, the following conditions hold:

$$
\sigma_{n}^{2}=\operatorname{Var} \xi_{n}<\infty \quad \text { and } \quad \sum_{n=1}^{\infty} \frac{\sigma_{n}^{2}}{n^{2}}<\infty
$$

Then the sequence $\left\{\xi_{n}, n \in \mathbb{N}\right\}$ satisfies $S L L N$ in the sense of Definition 2.

At the end of the section, we recall the following more important statement from [14, p. 359, Ch. IV, Sect. 2], which gives criteria that will determine whether a sum of independent random variables converges or diverges.

Theorem 4. (Kolmogorov and Khinchin). Let $\left\{\xi_{n}\right\}$ be the sequence of independent random variables.

(a) Let $G F \mathrm{E} \xi_{n}=0$ for all $n \in \mathbb{N}$. Then if

$$
\sum_{n=1}^{\infty} \mathrm{E} \xi_{n}^{2}<\infty
$$

the series $\sum_{n=1}^{\infty} \mathrm{E} \xi$ converges with Probability 1.

(b) If the random variables $\left\{\xi_{n}\right\}$ are uniformly bounded (i.e., $\left.\mathbb{P}\left(\left|\xi_{n}\right| \leq c\right)=1, c<\infty\right)$, the converse is true: the convergence of $\sum_{n=1}^{\infty} \mathrm{E} \xi$ with Probability 1 implies $\sum_{n=1}^{\infty} \mathrm{E} \xi_{n}^{2}<\infty$.

\subsection{Central limit theorem}

The solution of the Central limit problem allows us to describe the class of limit distribution functions for sums of independent random variables, when the contribution of each term is infinitely less than their sum. Under certain conditions, the sum of independent random variables, each of which is infinitesimal, has a distribution close to the normal distribution (the Gaussian distribution). The value of this result goes far beyond the scope of probability theory. It serves as the basis for using the normal distribution in the solution of many practical problems.

According to papers we give the following Definition and Theorem about the Central limit theorem; see [5], [6].

Let $(\boldsymbol{\Omega}, \mathcal{F}, \mathbb{P})$ be an arbitrary probability space and $\left\{\xi_{n}, n \in\right.$ $\mathbb{N}\}$ be a sequence of random variables defined on this space. And let $\mathbb{R}$ is the set of real numbers.

Definition 3. If for any $x \in \mathbb{R}$

$$
\mathbb{P}\left(\frac{S_{n}-\mathrm{E} S_{n}}{\sqrt{\operatorname{Var} S_{n}}} \leq x\right) \longrightarrow \Phi(x)
$$

where

$$
\Phi(x)=\frac{1}{\sqrt{2 \pi}} \int_{-\infty}^{x} e^{-u^{2} / 2} d u
$$

then the sequence of random variables $\left\{\xi_{n}, n \in \mathbb{N}\right\}$ is said to satisfy the Central limit theorem (CLT).
Under certain conditions, the Central limit theorem also holds for a sequence of random variables with different distributions. If for any $\tau>0$

$$
L_{\tau}(n):=\frac{1}{B_{n}^{2}} \sum_{k=1}^{n} \int_{\left|x-a_{k}\right|>\tau B_{n}}\left(x-a_{k}\right)^{2} d F_{k}(x) \longrightarrow 0
$$

as $n \rightarrow \infty$, then the sequence $\left\{\xi_{n}, n \in \mathbb{N}\right\}$ is said to satisfy the Lindeberg condition, where

$$
a_{k}=\mathrm{E} \xi_{k}, \quad B_{n}^{2}=\sum_{k=1}^{n} \operatorname{Var} \xi_{k}
$$

and $F_{k}(x)$ is the distribution function of the random variable $\xi_{k}$.

Theorem 5. (Lindeberg CLT). If for the sequence of random variables $\left\{\xi_{n}, n \in \mathbb{N}\right\}$, the Lindeberg condition (1.2) holds, then

$$
\mathbb{P}\left(\frac{S_{n}-\sum_{k=1}^{n} a_{k}}{B_{n}} \leq x\right) \longrightarrow \Phi(x) \text { as } n \rightarrow \infty
$$

for any $x \in \mathbb{R}$.

\section{Sums of random variables in a special form and asymptotes of their vari- ances}

Now let $\left\{\xi_{n}, n \in \mathbb{N}\right\}$ be a sequence of independent and identically distributed random variables with

$$
\mathrm{E} \xi_{1}=0 \text { and } \operatorname{Var} \xi_{1}=\sigma^{2}>0
$$

Denote

$$
S_{0}=0, \quad S_{n}=\sum_{i=1}^{n} \xi_{i}, \quad B_{n}^{2}=\operatorname{Var} S_{n}, \quad n \in \mathbb{N} .
$$

It is easy to see, that $\mathrm{E} S_{n}=0, B_{n}^{2}=\sigma^{2} n$.

Let $\xi_{1}^{(n)}, \xi_{2}^{(n)}, \ldots, \xi_{k}^{(n)}, \ldots$ be a sequence of independent and identically distributed random variables with

$$
\mathrm{E} \xi_{1}^{(n)}=0 \quad \text { and } \quad \operatorname{Var} \xi_{1}^{(n)}=\sigma_{n}^{2}>0
$$

Put

$$
S_{0}^{(n)}=0, \quad S_{n}^{(n)}=\sum_{i=1}^{n} \xi_{i}^{(n)}
$$

for all $n \in \mathbb{N}$.

Define the following sums of random variables of a special form:

$$
X_{n}=\sum_{k=1}^{n} m^{k} S_{k}, \quad Y_{n}=\sum_{k=1}^{n} m^{n-k} S_{k}
$$

for some $m>0$ and

$$
Z_{n}=\sum_{k=1}^{n} m_{n}^{k} S_{k}, \quad T_{n}=\sum_{k=1}^{n} m_{n}^{n-k} S_{k}
$$




$$
J_{n}^{(n)}=\sum_{k=1}^{n} m_{n}^{k} S_{k}^{(n)}, \quad K_{n}^{(n)}=\sum_{k=1}^{n} m_{n}^{n-k} S_{k}^{(n)},
$$

where

$$
m_{n}=1+\frac{\alpha}{n}+o\left(\frac{1}{n}\right) \quad \text { as } \quad n \rightarrow \infty
$$

for some $\alpha \in \mathbb{R} \backslash\{0\}$.

We calculate the mean and variance of these sums and we study the asymptotic behavior of variances.

At first, we can see

$$
\mathrm{E} X_{n}=\mathrm{E}\left(\sum_{k=1}^{n} m^{k} S_{k}\right)=\sum_{k=1}^{n} m^{k} \mathrm{E} S_{k}=0
$$

Similar to equality (2.1), it is easy to see that the mathematical expectations of the sums $Y_{n}, Z_{n}, T_{n}, J_{n}^{(n)}$ and $K_{n}^{(n)}$ are also equal to 0 .

To calculate the variance of random variable $X_{n}$ let's rewrite it as follows:

$$
X_{n}= \begin{cases}\sum_{i=1}^{n} \frac{m^{n+1}-m^{i}}{m-1} \xi_{i}, & \text { if } \quad m \neq 1, \\ \sum_{i=1}^{n}(n-i+1) \xi_{i} & , \quad \text { if } \quad m=1 .\end{cases}
$$

Now taking into account independence of random variables $\xi_{i}$ and relation 2.2], we can calculate the variance of $X_{n}$ :

$$
\begin{aligned}
& \operatorname{Var} X_{n}=\Sigma(m) \times \\
& \times\left[n m^{2 n+2}-2 \frac{m^{n+2}\left(m^{n}-1\right)}{m-1}+\frac{m^{2}\left(m^{2 n}-1\right)}{m^{2}-1}\right]
\end{aligned}
$$

if $m \neq 1$, where

$$
\Sigma(m):=\frac{\sigma^{2}}{(m-1)^{2}}
$$

and

$$
\operatorname{Var} X_{n}=\frac{n(n+1)(2 n+1)}{6} \sigma^{2}
$$

if $m=1$.

It is known, for $0 \leq m<1$, the relations

$$
\lim _{n \rightarrow \infty} m^{n}=0 \quad \text { and } \quad \lim _{n \rightarrow \infty} n m^{n}=0
$$

hold. Therefore, we obtain the following asymptotes for the variance of $X_{n}$ :

$$
\operatorname{Var} X_{n} \sim \begin{cases}\Sigma(m) \cdot \frac{m^{2}}{1-m^{2}}, & \text { if } \quad m<1, \\ \frac{n^{3}}{3} \sigma^{2}, & \text { if } \quad m=1, \\ \Sigma(m) \cdot n m^{2 n+2}, & \text { if } \quad m>1\end{cases}
$$

as $n \rightarrow \infty$.
To calculate the variance of $Y_{n}$, we rewrite it as follows:

$$
Y_{n}=\sum_{k=1}^{n} m^{n-k} S_{k}=\sum_{i=1}^{n} \frac{m^{n-i+1}-1}{m-1} \xi_{i} .
$$

Taking into account independence of random variables $\xi_{i}$ and relation 2.5) we obtain

$$
\operatorname{Var} Y_{n}=\Sigma(m) \cdot\left[\frac{m^{2}\left(m^{2 n}-1\right)}{m^{2}-1}-2 \frac{m\left(m^{n}-1\right)}{m-1}+n\right] .
$$

Due to the relations 2.3, it follows that

$$
\operatorname{Var} Y_{n} \sim \begin{cases}\Sigma(m) \cdot n, & \text { if } m<1, \\ \Sigma(m) \cdot \frac{m^{2 n+2}}{m^{2}-1}, & \text { if } \quad m>1\end{cases}
$$

as $n \rightarrow \infty$.

Similarly, one can rewrite the random variable $Z_{n}$ as follows:

$$
Z_{n}=\sum_{i=1}^{n} \frac{m_{n}^{n+1}-m_{n}^{i}}{m_{n}-1} \xi_{i}
$$

Taking into account independence of random variables $\xi_{i}$ and relation 2.7], we obtain

$$
\begin{aligned}
& \operatorname{Var} Z_{n}=\Sigma\left(m_{n}\right) \times \\
& \times\left[n m_{n}^{2 n+2}-2 \frac{m_{n}^{n+2}\left(m_{n}^{n}-1\right)}{m_{n}-1}+\frac{m_{n}^{2}\left(m_{n}^{2 n}-1\right)}{m_{n}^{2}-1}\right],
\end{aligned}
$$

where

$$
\Sigma\left(m_{n}\right):=\frac{\sigma^{2}}{\left(m_{n}-1\right)^{2}} .
$$

Since

$$
m_{n}=1+\frac{\alpha}{n}+o\left(\frac{1}{n}\right) \quad \text { as } \quad n \rightarrow \infty
$$

it is easy to see that

$$
\lim _{n \rightarrow \infty} m_{n}^{n}=e^{\alpha}
$$

Therefore,

$$
\operatorname{Var} Z_{n} \sim \frac{n^{3} \sigma^{2}}{2 \alpha^{3}} \cdot\left[e^{2 \alpha}(2 \alpha-3)+4 e^{\alpha}-1\right]
$$

as $n \rightarrow \infty$.

Further, rewrite $T_{n}$ as follows:

$$
T_{n}=\sum_{i=1}^{n} \frac{m_{n}^{n-i+1}-1}{m_{n}-1} \xi_{i} .
$$

Relation 2.10 gives

$$
\operatorname{Var} T_{n}=\Sigma\left(m_{n}\right)\left[\frac{m_{n}^{2}\left(m_{n}^{2 n}-1\right)}{m_{n}^{2}-1}-2 \frac{m_{n}\left(m_{n}^{n}-1\right)}{m_{n}-1}+n\right] .
$$

Using (2.8) we obtain asymptotic relation for the variance of $T_{n}$ :

$$
\operatorname{Var} T_{n} \sim \frac{n^{3} \sigma^{2}}{2 \alpha^{3}} \cdot\left(e^{2 \alpha}-4 e^{\alpha}+2 \alpha+3\right)
$$


as $n \rightarrow \infty$.

Now we can see

$$
J_{n}^{(n)}=\sum_{k=1}^{n} m_{n}^{k} S_{k}^{(n)}=\sum_{i=1}^{n} \frac{m_{n}^{n+1}-m_{n}^{i}}{m_{n}-1} \xi_{i}^{(n)}
$$

and taking into account independence of random variables $\xi_{i}^{(n)}$ we obtain

$$
\begin{aligned}
& \operatorname{Var} J_{n}^{(n)}=\Sigma_{n}\left(m_{n}\right) \times \\
& \times\left[n m_{n}^{2 n+2}-2 \frac{m_{n}^{n+2}\left(m_{n}^{n}-1\right)}{m_{n}-1}+\frac{m_{n}^{2}\left(m_{n}^{2 n}-1\right)}{m_{n}^{2}-1}\right],
\end{aligned}
$$

where

$$
\Sigma_{n}\left(m_{n}\right)=\frac{\sigma_{n}^{2}}{\left(m_{n}-1\right)^{2}} .
$$

Due to the relation 2.8 , the following asymptotic relation follows:

$$
\operatorname{Var} J_{n}^{(n)} \sim \frac{n^{3} \sigma_{n}^{2}}{2 \alpha^{3}} \cdot\left[e^{2 \alpha}(2 \alpha-3)+4 e^{\alpha}-1\right]
$$

as $n \rightarrow \infty$.

Finally, we rewrite the random variable $K_{n}^{(n)}$ as follows:

$$
K_{n}^{(n)}=\sum_{i=1}^{n} \frac{m_{n}^{n-i+1}-1}{m_{n}-1} \xi_{i}^{(n)}
$$

and calculate the variance of $K_{n}^{(n)}$ :

$$
\begin{aligned}
& \operatorname{Var} K_{n}^{(n)}=\Sigma_{n}\left(m_{n}\right) \times \\
& \times\left[\frac{m_{n}^{2}\left(m_{n}^{2 n}-1\right)}{m_{n}^{2}-1}-2 m_{n} \frac{m_{n}^{n}-1}{m_{n}-1}+n\right] .
\end{aligned}
$$

Formula 2.8 yields the following asymptote:

$$
\operatorname{Var} K_{n}^{(n)} \sim \frac{n^{3} \sigma_{n}^{2}}{2 \alpha^{3}} \cdot\left(e^{2 \alpha}-4 e^{\alpha}+2 \alpha+3\right)
$$

as $n \rightarrow \infty$.

Let's now check if the above defined special form sums satisfy LLN.

For the random variable $X_{n}$, it follows from (2.4) that

$$
\lim _{n \rightarrow \infty} \frac{1}{n^{2}} \cdot \operatorname{Var} X_{n}= \begin{cases}0, & \text { if } m<1, \\ \infty, & \text { otherwise. }\end{cases}
$$

Relation 2.14 implies that the sum $X_{n}$ satisfies LLN if $m<1$.

Relation (2.6) implies that

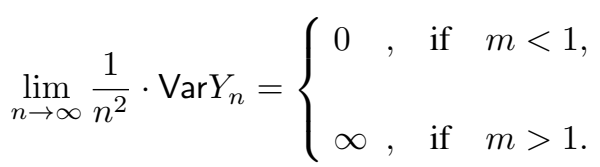

So that the sum $Y_{n}$ satisfies LLN if $m<1$.

From relations 2.9, 2.11)-2.13) follows that

$$
\begin{gathered}
\lim _{n \rightarrow \infty} \frac{1}{n^{2}} \cdot \operatorname{Var} Z_{n}=\lim _{n \rightarrow \infty} \frac{1}{n^{2}} \cdot \operatorname{Var} T_{n}=\infty, \\
\lim _{n \rightarrow \infty} \frac{1}{n^{2}} \cdot \operatorname{Var} J_{n}^{(n)}=\lim _{n \rightarrow \infty} \frac{1}{n^{2}} \cdot \operatorname{Var} K_{n}^{(n)}=\infty .
\end{gathered}
$$

Thus, these variables do not satisfy LLN.

\section{Central limit theorem analogues}

The theorems below reveal the asymptotic properties of the sums $X_{n}, Y_{n}, Z_{n}, T_{n}, J_{n}^{(n)}$ and $K_{n}^{(n)}$ as $n \rightarrow \infty$.

\section{Theorem 6. The following assertions hold:}

(i) for $m<1$ the sequence of random variables $X_{n}$ will converge with probability one;

(ii) for $m>1$

$$
\mathbb{P}\left(\frac{(m-1) \cdot X_{n}}{\sigma m^{n+1} \sqrt{n}} \leq x\right) \longrightarrow \Phi(x) \text { as } n \rightarrow \infty
$$

(iii) for $m=1$

$$
\mathbb{P}\left(\frac{\sqrt{3} \cdot X_{n}}{\sigma n \sqrt{n}} \leq x\right) \longrightarrow \Phi(x) \text { as } n \rightarrow \infty .
$$

The following result follows from Theorem 6 .

Corollary 1. The following assertions hold:

(i) for $m<1$

$$
\mathbb{P}\left(\frac{(m-1) \cdot Y_{n}}{\sigma \sqrt{n}} \leq x\right) \longrightarrow \Phi(x) \text { as } n \rightarrow \infty
$$

(ii) for $m>1$

$$
\mathbb{P}\left(\frac{(m-1) \cdot Y_{n}}{\sigma m^{n+1} \sqrt{m^{2}-1}} \leq x\right) \longrightarrow \Phi(x) \quad \text { as } \quad n \rightarrow \infty .
$$

Remark 1. We omit the case $m=1$, since $Y_{n}=X_{n}$ in this case, Part (3) of Theorem 6 holds identically.

Theorem 7. The following assertions hold:

$$
\begin{aligned}
& \mathbb{P}\left(\frac{\alpha \sqrt{2 \alpha} \cdot Z_{n}}{\sigma n \sqrt{n}} \leq x\right) \longrightarrow \Phi(x) \text { as } n \rightarrow \infty ; \\
& \mathbb{P}\left(\frac{\alpha \sqrt{2 \alpha} \cdot T_{n}}{\sigma n \sqrt{n}} \leq x\right) \longrightarrow \Phi(x) \text { as } n \rightarrow \infty .
\end{aligned}
$$

The following result follows from the Theorem 7

Corollary 2. The following assertions hold:

$$
\begin{aligned}
& \mathbb{P}\left(\frac{\alpha \sqrt{2 \alpha} \cdot J_{n}^{(n)}}{\sigma_{n} n \sqrt{n}} \leq x\right) \longrightarrow \Phi(x) \text { as } n \rightarrow \infty \\
& \mathbb{P}\left(\frac{\alpha \sqrt{2 \alpha} \cdot K_{n}^{(n)}}{\sigma_{n} n \sqrt{n}} \leq x\right) \longrightarrow \Phi(x) \text { as } n \rightarrow \infty .
\end{aligned}
$$




\section{Proof of Theorems}

Proof of Theorem 6. For the case $m<1$ we recall

$$
X_{n}=\sum_{i=1}^{n} x_{i}
$$

where

$$
x_{i}:=\frac{m^{n+1}-m^{i}}{m-1} \xi_{i} .
$$

Since $\xi_{i}$ are independent variables, $x_{i}$ are also independent variables. It follows from (2.4) that

$$
\lim _{n \rightarrow \infty} \sum_{i=1}^{n} \operatorname{Var} x_{i}<\infty
$$

Then according to the Theorem 4 the sequence $\left\{X_{n}\right\}$ converges with probability one.

The Part (i) is proved.

Now for $m>1$ we define

$$
F_{i}(x):=\mathbb{P}\left(x_{i}<x\right) .
$$

Then

$$
F_{i}(x)=F\left(\frac{m-1}{m^{n+1}-m^{i}} x\right),
$$

where

$$
F(x)=\mathbb{P}\left(\xi_{1}<x\right) .
$$

We check the random variable $X_{n}$ for the fulfillment of condition (1.2). Write the Lindeberg function for the sum $X_{n}=\sum_{i=1}^{n} x_{i}$. We have $\mathrm{E} x_{i}=0$ since $\mathrm{E} \xi_{i}=0$. Then

$$
L_{\varepsilon}(n)=\frac{1}{B_{n}^{2}} \sum_{i=1}^{n} \int_{|x|>\varepsilon B_{n}} x^{2} d F_{i}(x)
$$

for any $\varepsilon>0$, where

$$
B_{n}^{2}:=\operatorname{Var} X_{n}=\sum_{i=1}^{n} \int_{\mathbb{R}} x^{2} d F_{i}(x) .
$$

According to relation (4.2), the Lindeberg function can be written in the following form:

$$
\begin{aligned}
L_{\varepsilon}(n) & =\frac{1}{B_{n}^{2}} \sum_{i=1}^{n} \int_{|x|>\varepsilon B_{n}} x^{2} d F\left(\frac{m-1}{m^{n+1}-m^{i}} x\right) \\
& =\frac{1}{B_{n}^{2}} \sum_{i=1}^{n}\left(\frac{m^{n+1}-m^{i}}{m-1}\right)^{2} \int_{I_{n}(i)} y^{2} d F(y),
\end{aligned}
$$

where

$$
I_{n}(i):=\left\{|y|>\frac{m-1}{m^{n+1}-m^{i}} \varepsilon B_{n}\right\} .
$$

Now let's estimate the last integral. So we see

$$
\int_{I_{n}(i)} y^{2} d F(y) \leq \int_{I_{n}(1)} y^{2} d F(y) .
$$

Due to the relation 2.4 for $m>1$,

$$
\lim _{n \rightarrow \infty} \frac{m-1}{m\left(m^{n}-1\right)} \varepsilon B_{n}=\infty .
$$

Hence, it follows that

$$
\int_{I_{n}(1)} y^{2} d F(y) \longrightarrow 0 \text { as } n \rightarrow \infty
$$

since

$$
\sigma^{2}=\int_{\mathbb{R}} y^{2} d F(y)<\infty .
$$

It follows from 4.1 that

$$
\frac{1}{B_{n}^{2}} \sum_{i=1}^{n}\left(\frac{m^{n+1}-m^{i}}{m-1}\right)^{2}=\frac{1}{\sigma^{2}} .
$$

Due to this, and with considering 4.3, we see that the Lindeberg condition (1.2) is fulfilled.

Therefore, the Part (ii) is proved according to CLT for independent random variables.

Now for the case $m=1$ we recall

$$
X_{n}=\sum_{i=1}^{n}(n-i+1) \xi_{i}
$$

Defining $z_{i}:=(n-i+1) \xi_{i}$, we have

$$
F_{i}(x):=\mathbb{P}\left(z_{i}<x\right)=F\left(\frac{x}{n-i+1}\right),
$$

where $F(x):=\mathbb{P}\left(\xi_{1}<x\right)$. Then the Lindeberg function is

$$
L_{\varepsilon}(n)=\frac{1}{B_{n}^{2}} \sum_{i=1}^{n}(n-i+1)^{2} \int_{J_{n}(i)} t^{2} d F(t),
$$

where $B_{n}^{2}=\operatorname{Var} X_{n}$ and

$$
J_{n}(i):=\left\{|t|>\frac{\varepsilon B_{n}}{n-i+1}\right\}
$$

for any $\varepsilon>0$.

We easily make sure that

$$
\int_{J_{n}(i)} t^{2} d F(t) \leq \int_{J_{n}(1)} t^{2} d F(t) .
$$

Due to the relation (2.4) for $m=1$, it follows that

$$
\frac{\varepsilon B_{n}}{n} \longrightarrow \infty \quad \text { as } \quad n \rightarrow \infty .
$$

Therefore, it follows that

$$
\int_{J_{n}(1)} y^{2} d F(y) \longrightarrow 0 \quad \text { as } \quad n \rightarrow \infty
$$

since

$$
\sigma^{2}=\int_{\mathbb{R}} y^{2} d F(y)<\infty .
$$


It is convincing that

$$
\frac{1}{B_{n}^{2}} \sum_{i=1}^{n}(n-i+1)^{2}=\frac{1}{\sigma^{2}} .
$$

Then the fulfillment of the Lindeberg condition follows from relations (4.4) and (4.5).

So the Part (iii) is proved according to CLT for independent random variables.

The Theorem 6 is proved completely.

Proof of Theorem 7 . In accordance with formula 2.7), we need a distribution function of the random variable

$$
\eta_{i}:=\frac{m_{n}^{n+1}-m_{n}^{i}}{m_{n}-1} \xi_{i}
$$

which is

$$
\mathbb{P}\left(\eta_{i}<x\right)=F\left(\frac{m_{n}-1}{m_{n}^{n+1}-m_{n}^{i}} x\right) .
$$

Then we can write the Lindeberg function as follows:

$$
L_{\varepsilon}(n)=\frac{1}{\operatorname{Var} Z_{n}} \sum_{i=1}^{n}\left(\frac{m_{n}^{n+1}-m_{n}^{i}}{m_{n}-1}\right)^{2} \int_{\mathcal{J}_{n}(i)} y^{2} d F(y),
$$

where

$$
\mathcal{J}_{n}(i)=\left\{|y|>\frac{m_{n}-1}{m_{n}^{n+1}-m_{n}^{i}} \varepsilon \operatorname{Var} Z_{n}\right\}
$$

for any $\varepsilon>0$.

As before we can see, that

$$
\int_{\mathcal{J}_{n}(i)} y^{2} d F(y) \leq \int_{\mathcal{J}_{n}(1)} y^{2} d F(y) .
$$

Decidedly $Z_{n} \geq Z_{1}=m_{n} S_{1}>\xi_{1}$ and thus $\operatorname{Var} Z_{n}>\sigma^{2}$. Hence we obtain

$$
L_{\varepsilon}(n) \leq \frac{1}{\sigma^{2}} \cdot \int_{\mathcal{J}_{n}(1)} y^{2} d F(y) .
$$

On the other hand it follows from 2.8 and 2.9 that

$$
\lim _{n \rightarrow \infty} \frac{m_{n}-1}{m_{n}^{n+1}-m_{n}} \varepsilon \operatorname{Var} Z_{n}=\infty .
$$

Therefore

$$
\int_{\mathcal{J}_{n}(1)} y^{2} d F(y) \longrightarrow 0 \quad \text { as } n \rightarrow \infty
$$

since

$$
\sigma^{2}=\int_{\mathbb{R}} y^{2} d F(y)<\infty .
$$

Now the fulfillment of the Lindeberg condition follows from relations 4.6 and 4.7.

$$
\frac{1}{\operatorname{Var} Z_{n}} \sum_{i=1}^{n}\left(\frac{m_{n}^{n+1}-m_{n}^{i}}{m_{n}-1}\right)^{2}=\frac{1}{\sigma^{2}} .
$$

The Part (i) is proved according to CLT for independent random variables.

The part of the theorem on $T_{n}$ is proved in a same way.

\section{REFERENCES}

[1] Imomov A.A. On long-time behaviors of states of GaltonWatson branching processes allowing immigration. Jour. of Siberian Fed. Univ.: Math. and Physics, 2015, vol. 8(4), 394405.

[2] Imomov A.A. Limit theorem for the joint distribution in the Q-processes. Jour. of Siberian Fed. Univ.: Math. and Physics, 2014, vol. 7(3), 289-296.

[3] Ispany M., Pap G., M.C.A. Van Zuijlen. Asymptotic Inference for Nearly Unstable INAR(1) Models. Jour. Appl. Prob. 2003, vol. 40(3), 750-765.

[4] Ispany M., Pap G., M.C.A. Van Zuijlen. Fluctuation limit of branching processes with immigration and estimation of the means. Adv. Appl. Prob. 2005, vol. 37, 523-538.

[5] Borovkov A. A. Teoriya veroyatnostey. URSS, Moscow, 2009 (in Russan).

[6] Feller W. An Introduction to Probability Theory and its Applications, vol. 1. John Wiley \& Sons, 1968.

[7] Kharin Yu, Zuyev N, Jook E. Teoriya veroyatnostey, matematicheskaya i prikladnaya statistika. Minsk, Belarus univ. Press, 2011 (in Russan).

[8] Petrov V. V. One-sided law of large numbers for ruled sums. Vestnik Leningrad. Univ., 1974, 55-59.

[9] Heyde C. C. A pair of complementary theorems on convergence rates in the law of large numbers. Proc. Camb. Phil. Soc. 1967, vol. 63(1), 73-82.

[10] Hsu P. L., Robbins H. Complete convergence and the law of large numbers. Proc. Nat. Acad. Sci. USA. 1947, vol. 33(2), 25-31.

[11] Bennett G. Probability inequalities for the sum of independent random variables. Journ. Amer Statist. Assoc. 1962, vol. 57, 3345.

[12] Hatori H., Maejima M., Mori T. Convergence rates in the law of large numbers when extreme terms are excluded. Z.Wahrscheinlichketstheorie verw., Gebiete. 1979, vol. 47, 112.

[13] Mori T. The strong law of large numbers when extreme terms are excluded from sums. Z.Wahrscheinlichketstheorie verw., Gebiete. 1976, vol. 36, 189-194.

[14] Shiryayev A. N. Probability, Springer-Verlag New York, Inc., 1984. 